\title{
Laparoscopic Evaluation of Chronic Pelvic Pain in Women
}

\author{
Chhetri S, ${ }^{1}$ Khanna $S,{ }^{1}$ Poonam, ${ }^{1}$ Sen $B^{1}$ \\ ${ }^{1}$ Department of Obstetrics and Gynecology, B.P. Koirala Institute of Health Sciences, Dharan Nepal.
}

\begin{abstract}
Background: Chronic pelvic pain is a common and significant disorder of women. Often the etiology of chronic pelvic pain is not clear.

Methods: A descriptive hospital based study was done which included 55 women with chronic pelvic pain of more than six months duration. Ultrasonography and diagnostic laparoscopy were performed in all patients. These patients also had a psychological assessment with the SRQ (Self Reporting Questionnaire). The various causes of pelvic pain were assessed.

Result: The mean age of women presenting with chronic pelvic pain was $37.1 \pm 2.3$ years. Ultrasonography detected abnormality in only $21(38.2 \%)$ patients. The most common pathology detected was ovarian cysts in seven patients. Diagnostic laparoscopy was able to detect pathology in $45(81.8 \%)$ patients. Pelvic adhesions was the most common cause of chronic pelvic pain which was present in $16(29 \%)$ of the women, followed by PID (pelvic inflammatory disease) in seven $(12.7 \%)$, endometriosis in five $(9.1 \%)$, pelvic congestion in four $(7.2 \%)$, pelvic tuberculosis in four $(7.2 \%)$, fibroid uterus in four $(7.2 \%)$, ovarian cysts in four $(7.2 \%)$ and parafimbrial cyst in one patient. No abnormality on laparoscopy was detected in $10(18.2 \%)$ patients. When evaluated for associated psychological illness it was detected in $24(43.6 \%)$ patients.

Results: Chronic pelvic pain is a common problem and presents a major challenge to health care providers because of its unclear etiology, complex natural history, and poor response to therapy. Diagnostic laparoscopy is thus an important tool in the evaluation of women with chronic pelvic pain.

Key words: chronic pelvic pain, laparoscopy, ultrasonography.
\end{abstract}

\section{INTRODUCTION}

Chronic pelvic pain is defined as a non - cyclic pelvic pain of greater than six months, which is not relieved by nonnarcotic analgesics. ${ }^{1}$ Chronic pelvic pain has been found to be one of the most common symptoms attending gynecological outpatient department in gynecology. Prevalence of chronic pelvic pain has been reported as $3.8 \%$ in women aged 15 - 73, which is higher than the prevalence of migraine $(2.1 \%)$ and similar to that of bronchial asthma (3.7\%) or back pain $(4.1 \%) .{ }^{2}$ in primary care practices, $39 \%$ of women complain of pelvic pain. ${ }^{3}$ Chronic pelvic pain is estimated to account for $10 \%$ of all referrals to gynecologists, it is the indication for $12 \%$ of all hysterectomies and over $40 \%$ of gynecologic laparoscopies. ${ }^{1}$ Clearly, pelvic pain is an important issue in the health care of women. Chronic pelvic pain seem to occur during reproductive years although some particular enigmatic pain syndromes may occur in young adolescents and other causes may occur after menopause and in the elderly.

Correspondence: Dr. Shailaja Chhetri, Department of Obstetrics and Gynecology, B.P. Koirala Institute of Health Sciences, Dharan Nepal. Phone: 9842063421, Email: shailzac@hotmail.com 
Laparoscopic Evaluation of Chronic Pelvic Pain in Women

Chronic pelvic pain is characterized by six common characteristics; duration of six months or longer, incomplete relief by previous treatment, pain out of proportion to tissue damage, loss of physical function at home or work, vegetative signs of depression and altered family and social roles.

Laparoscopy is a valuable clinical tool in the diagnosis of chronic pelvic pain. It can confirm a clinical impression, establish a definite diagnosis, follow the course of a disease and modify therapy accordingly.

\section{METHODS}

It was a descriptive hospital based study that was conducted in the department of Obstetrics and Gynecology in association with the department of Psychiatry of B. P. Koirala Institute of Health Sciences, Dharan which is a tertiary referral centre which provides health care facilities to the people of eastern Nepal, from January 2004 to May 2005. The main aim of the study was to assess the various causes of chronic pelvic pain with the help of laparoscopy as a diagnostic tool and to ascertain the role played by psychological factors in these women.

Fifty-five women attending the gynecological out patients department with pelvic pain of more than six months duration in spite of treatment were included in the study.

Patients were excluded from the study if they had nongynecological causes of chronic pelvic pain.

Informed consent was obtained from the subjects and the hospital ethical board committee ethically approved the study.

All subjects had a detailed history and examination performed and all of them underwent an ultrasonogram of the abdomen and pelvis. Diagnostic laparoscopies were performed on all fifty patients.

Assessment of psychological factors were done using the SRQ-20 (Self Reporting Questionnaire). This questionnaire has been developed by the WHO as an instrument designed to screen for psychiatric disturbance especially in developing countries. It may be used as a selfadministered or as an interviewer-administered questionnaire. The maximum score is 20. A score of more than or equal to eight suggests that the patient may have a probable psychiatric element.

All observations will be recorded by interview method. All data were analyzed systematically.

\section{RESULTS}

A total of 73 patients were initially screened for the study. Eighteen were excluded as 11 of them had irritable bowel syndrome, four had cystitis, and three had chronic appendicitis. Fifty-five women were thus included in the study.

The mean age of patients with chronic pelvic pain was $37.1 \pm 2.3$ years. Chronic pelvic pain was found more among multiparous women, majority having parity two (34\%). Infertility was present in $10 \%$ of women. The mean duration of pain was $3.93 \pm 0.71$ years.

Majority of the patients (76\%) had some associated symptoms with chronic pelvic pain. The most common was vaginal discharge, which was present in $36 \%$ of cases, followed by dysparuenia in $32 \%$. Menstrual abnormalities were present in $28 \%$, dysmenorrhoea in $24 \%$ and sleep disturbances in $18 \%$.

Physical examination revealed abdominal tenderness in $44 \%$ of patients with chronic pelvic pain and, cervical motion tenderness was noted in $36 \%$ of patients. Bulky uterus was detected in $12 \%$ of patients and adnexal mass and restricted mobility in $8 \%$ each. No physical abnormality on examination was present in $10 \%$ of patients.

Eighteen patients (32.7\%) had history of a previous abdominal surgery. The most common prior surgical procedure preformed in patients with chronic pelvic pain was tubal ligation, which was performed in seven patients (14\%), followed by appendicectomy in three patients $(6 \%)$ and hysterectomy in three patients $(6 \%)$. Dilatation and curettage was performed in two patients, ovarian cystectomy, caesarean section and cholecystectomy in one patient each.

Thirty-four patients (61.8\%) had normal ultrasonography findings. An abnormal ultrasonogram was detected in only 21 of the patients with chronic pelvic pain. Seven (12.7\%) of patients had cystic ovaries and six (10.9\%) patients had PID (Pelvic Inflammatory Disease). Uterine fibroids were detected in three $(5.4 \%)$ cases, bulky uterus in three $(5.4 \%)$ cases and adenexal mass in two (3.6\%) of patients with chronic pelvic pain.

Diagnostic laparoscopy detected abnormalities in 45 $(81.8 \%)$ of the patients with chronic pelvic pain. Pelvic adhesion was the most common etiology diagnosed by laparoscopy and was present in $16(29 \%)$ of the patients, while PID (Pelvic Inflammatory Disease) was found in 7 $(12.7 \%)$ of the patients. Endometriosis was present in $5(9.1 \%)$, pelvic tuberculosis in $4(7.2 \%)$, pelvic congestion in $4(7.2 \%)$, fibroid uterus in $4(7.2 \%)$ and ovarian cysts in 
4 (7.2\%) respectively. Parafimbrial cyst was detected in one patient. Laparoscopy did not yield any diagnosis in $10(18.2 \%)$ patients (Figure 1$)$.

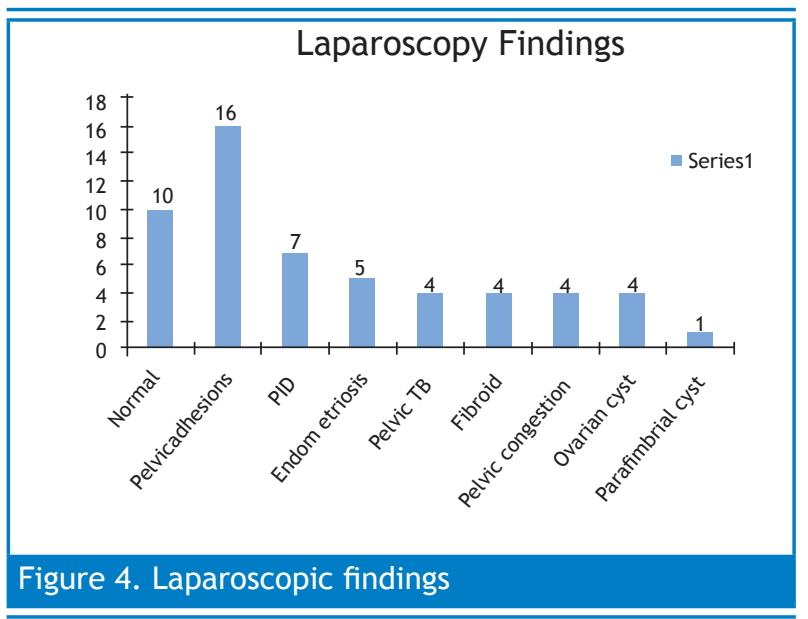

Among the 55 patients who were evaluated for chronic pelvic pain, $24(43.6 \%)$ patients had a SRQ score of $\geq 8$ which may indicate a probable psychiatric illness. Nineteen of these patients had an abnormal laparoscopic findings and the other five had no abnormality detected on laparoscopy.

\section{DISCUSSION}

The results of research on chronic pelvic pain must be reviewed carefully before the data can be generalized to primary care populations. Few studies have evaluated patients with chronic pelvic pain who present to family physicians. Much of the research has been performed on well-delineated referral populations; in women who were treated by gynecologists, in women who were treated in clinics for chronic pain in general or chronic pelvic pain specifically and in women with chronic pelvic pain that persisted despite hysterectomy. Women with chronic pelvic pain responsive to simpler and less invasive treatment seldom have been participants in these studies. Chronic pelvic pain has a myriad of possible causes, most with very little correlation between clinical evidence and the extent of disease and the quality, quantity or appearance of pain. Often, the woman with chronic pelvic pain has seen several physicians, has used alternative methods of care, may have already had a hysterectomy, may have used narcotics for pain relief and usually has multiple other complaints that span several organ systems, including dyspareunia, anorgasmy, post - coital pain,

disturbances in menstrual cycle, backache, nausea, malaise, diarrhoea, headaches and vertigo.

Chronic pelvic pain is predominant in the reproductive age group and the mean age of patients in our study was $37.08 \pm 7.65$ years, which was similar to the study performed by Zondervan et al in the United Kingdom. ${ }^{4}$

Ultrasonography, which is routinely performed in patients with chronic pelvic pain, did not detect any pathology in $62 \%$ of the patients. Pelvic adhesions, subtle changes such as minimal endometriosis and varicosities are however difficult to detect on an ultrasonogram.

Laparoscopy, because of its availability and safety is a valuable tool in the evaluation of undiagnosed chronic pelvic pain. It is a simple and often definitive means of establishing the presence or absence of pelvic pathology without resorting to major abdominal surgery. More than $40 \%$ of all laparoscopies are performed for the diagnosis of chronic pelvic pain. ${ }^{5}$ In the present study, diagnostic laparoscopy was performed in all 50 patients. Abnormal laparoscopy findings were detected in $80 \%$ of the patients while $20 \%$ patients had no abnormality detected. Adhesions were detected in $32 \%$ accounted for the most common pathology. Endometriosis were detected in $10 \%$ of the patients.

In similar studies done by Cunanan et al, Kresch et al, Marana et al, Kantoravdis et al and Mara et al, diagnostic laparoscopy detected pathology in $82.5 \%, 83 \%, 80 \%, 76 \%$ and $82.3 \%$ respectively. ${ }^{6-10}$ It was also noted that in these studies the most common pathology detected were pelvic adhesions.

Psychological evaluation is necessary in the initial evaluation and treatment of all women with chronic pelvic pain. Even when there is no suspicion of a psychologic diagnosis, it helps provide the doctor with specific information about the patient that may be related to therapeutic responsiveness and prognosis, and can be used in treatment planning, however this may not always be possible. Thus simple questionnaires like the SRQ maybe helpful.

The limitation of the study was that the data might not be attributed to the community, as the sample size was not large. Conscious laparoscopic pain mapping under local anesthesia was not performed which may have helped to identify subtle or atypical areas that might have been overlooked by laparoscopy.

\section{CONCLUSION}

Chronic pelvic pain is a common problem and presents a major challenge to health care providers because of its unclear etiology, complex natural history, and poor response to therapy.

Laparoscopy has a major role in the gynecologist's evaluation of potential visceral causes of chronic pelvic pain. Significant judgment is required in individualizing each woman's evaluation. Missing treatable disease 
Laparoscopic Evaluation of Chronic Pelvic Pain in Women

prolongs the patient's suffering and may even be life threatening in rare cases. In patients who have undergone laparoscopy, reassurance derived by the procedure may lead to improvement in their symptoms. Therefore laparoscopy has an important role in the diagnosis and treatment of patients with chronic pelvic pain and should be done in all patients with chronic pelvic pain.

\section{REFERENCES}

1. Howard FM. The role of laparoscopy in chronic pelvic pain: Promises and pitfalls. Obstet Gynecol Surv 1993; 48(6): 357 387.

2. Zondervan KT, Yudkin PL, Vessey MP, Dawes MG, Barlow DH, Kennedy SH. Prevalence and incidence in primary care of chronic pelvic pain in women: Evidence from a national general practice database. Br J Obstet Gynaecol 1999;106:1149-1155.

3. Jamieson DJ, Steege JF: The prevalence of dysmenorrhea, dyspareunia, pelvic pain, and irritable bowel syndrome in primary care practices. Obstet Gynecol 1996: 87(1): 55-58.

4. Zondervan ET, Yudkin PL, Vessey MP, Jenkinson CP, Dawes MG,
Barlow DH, Kennedy SH. Chronic pelvic pain in the community - symptoms, investigations, and diagnoses. Am J Obstet Gynecol 2001;184:1149-55.

5. Howard FM. The role of laparoscopy as a diagnostic tool in chronic pelvic pain. Ball Clin Obstet Gynecol 2000: 14(3); 467-494.

6. Cunanan RG, Norman MD, Courey NG, and Lippes J: Laparoscopic findings in patients with pelvic pain. Am J Obstet Gynecol 1983;146:589-591.

7. Kresch AJ, Seifer DB, Sachs LB, and Barrese I. Laparoscopy in 100 women with chronic pelvic pain. Obstet Gynecol 1984;64:672 674.

8. Marana R, Paielle FV, Muzii L, Dell'Acqua S, Mancuso S., : The role of laparoscopy in the evaluation of Chronic Pelvic Pain: Minerva Ginecol 1993:45(6);281-6.

9. Kontoravdis A, Chryssikopoulos A, Hassiakos D, Liapis A, Zourlas PA, The diagnostic value of laparoscopy in 2365 patients with acute and chronic pelvic pain; Int J Gynecol Obstet 1996:52(3);243-8.

10. Mara M, Fucikova Z, Kuzel D, Dohnalova A, Haakova L, Zivny J., Laparoscopy in chronic pelvic pain- a retrospective clinical study: Ceska gynekol $2002: 67(1): 38-46$. 